\title{
Tuning the Shape and Strain in Micro/Nanowires by a Sideways Physical Deposition Process
}

\author{
Yue Shen, ${ }^{\dagger, \star}$ Jung-Il Hong, ${ }^{\ddagger}$ Zhengchun Peng, ${ }^{\S}$ Hao Fang, ${ }^{\ddagger}$ Su Zhang, ${ }^{\dagger}$ Shuxiang Dong, ${ }^{\dagger}$ \\ Robert L. Snyder, ${ }^{\ddagger}$ and Zhong Lin Wang*, \\ Department of Advanced Materials and Nanotechnology, College of Engineering, Peking University, \\ Beijing 100871, People's Republic of China, and School of Materials Science and Engineering and School of \\ Mechanical Engineering, Georgia Institute of Technology, Atlanta, Georgia, 30332, United States
}

Received: September 12, 2010; Revised Manuscript Received: October 30, 2010

\begin{abstract}
We studied the bending phenomenon of $\mathrm{ZnO}$ nanowires (NWs) by using three kinds of sideways physical deposition processes: laser deposition (PLD), sputtering, and evaporation. Room temperature PLD is the most efficient method to bend NWs. The bending is probably caused by the ion implantation into the surface layers. Temperature can influence the bending angle at low temperatures (below $120^{\circ} \mathrm{C}$ ). The shape-modified NWs may have applications in nanoelectronics, optics, and surface wettability tuning.
\end{abstract}

\section{Introduction}

Engineering the shape and strain of nanostructures is of great importance to the fabrication and understanding of nanodevices. ${ }^{1-6}$ A lot of work has been done to grow useful nanomaterials with various morphologies such as nanowires (NWs), ${ }^{7,8}$ nanotubes, ${ }^{9}$ nanobelts, ${ }^{10}$ nanohelices,,${ }^{11,12}$ nanorings,${ }^{13}$ and nanotetrapods. ${ }^{14}$ Beside these as-grown nanostructures, research has been done to modify the shape of the nanostructrure postgrowth. For example, by changing straight NW arrays into bent NW arrays, the surface wettability has been modified. ${ }^{15,16}$ Strain results in a large change in the electrical transport properties of $\mathrm{ZnO}$ $\mathrm{NWs}^{17,18}$ and the near band gap emission, ${ }^{19,20}$ which is the piezotronic effect. Strain can also introduce a metal-insulator phase transition in $\mathrm{VO}_{2}{ }^{21,22}$

In our previous report, ${ }^{23}$ sideways pulsed laser deposition (PLD) was found to be a very effective method to fabricate arc-shaped composite NWs with uniform bending direction and bending angle/shape. Vertically aligned straight $\mathrm{ZnO}$ NWs were found to be bent after depositing any material on one side of the NWs. Considering that all of the NWs were bent to the same direction regardless of what material was deposited, the bending phenomenon cannot be explained by the difference of the thermal expansion coefficients or interfacial lattice mismatch. In this paper, we compared the bending phenomenon at different temperatures, and using different deposition methods, a possible formation mechanism of the bent NWs is proposed.

\section{Experimental Section}

The influence of temperature on the $\mathrm{ZnO} \mathrm{NWs}$ ' bending phenomenon in the sideways PLD process was studied. The original randomly dispersed $\mathrm{ZnO}$ NWs (Figure $1 \mathrm{~b}$ ) were grown on an $\mathrm{Au}$ substrate $(3 \mathrm{~mm} \times 3 \mathrm{~mm})$ using zinc nitrite and hexamethylenetetramine (HMTA) $2 \mathrm{mM} \mathrm{1:1} \mathrm{solution} \mathrm{at} 80^{\circ} \mathrm{C}$ for $16 \mathrm{~h} .{ }^{24}$ Then the sideways PLD process was carried out in a vacuum chamber (pressure $<2 \times 10^{-6}$ Torr) using the same

\footnotetext{
* Corresponding author. E-mail: zlwang@gatech.edu.

${ }^{\dagger}$ Peking University.

* School of Materials Science and Engineering, Georgia Institute of Technology.

${ }^{\S}$ School of Mechanical Engineering, Georgia Institute of Technology.
}

experimental parameters (Coherent Complex $205 \mathrm{KrF}$ laser, energy per pulse $=150 \mathrm{~mJ}$, repeat rate $=10 \mathrm{~Hz}$, deposition time $=1 \mathrm{~h}$, Figure 1a) at different temperatures. The fabricated samples were characterized using Leo 1550 scanning electron microscopy (SEM) at an acceleration voltage of $5 \mathrm{kV}$ and a PAnalytical X'pert Pro MRD X-ray diffraction system.

Different sideways deposition methods (PLD, sputter, and E-beam evaporator) were also studied for comparison purpose (Figure 3). High aspect ratio, freestanding $\mathrm{ZnO}$ microwires (synthesized by a physical deposition method, diameter $\approx 2$ $\mu \mathrm{m}$ and length $\approx 600 \mu \mathrm{m}$ ) were fixed at the edge of silicon wafers using a silver paste. Then, $200 \mathrm{~nm}$ Au was deposited from the top of these microwires using different methods. For PLD, a Coherent Complex $205 \mathrm{KrF}$ excimer laser was used. The energy per pulse, repeat rate, substrate temperature, and deposition time were set as $200 \mathrm{~mJ}, 10 \mathrm{~Hz}, 28{ }^{\circ} \mathrm{C}, 1.5 \mathrm{~h}$, respectively. For sputter, a Torr Magnetron sputtering system was used. The RF power and the Ar pressure were set as 120 $\mathrm{W}$ and 5 mTorr, respectively. The deposition rate was 0.2 $\mathrm{nm} \cdot \mathrm{s}^{-1}$. For E-beam evaporator, a CVC electron beam evaporator was used. The deposition rate was set as $0.3 \mathrm{~nm} \cdot \mathrm{s}^{-1}$. The same recipe was used to deposit $150 \mathrm{~nm}$ titanium on the side wall of vertically aligned silicon NWs. The silicon NWs were synthesized via a top-down deep reactive ion etching process. ${ }^{16,25}$ A $100 \mathrm{~nm}$ negative resist (hydrogen silsequioxane) was spincoated on a $\mathrm{Si}(100)$ wafer. A JC Nabity E-beam lithography system was used to write a square array of $250 \mathrm{~nm}$ dots. The distance between these dots was $5 \mu \mathrm{m}$. Then a Bosch process was carried out to etch down $5 \mu \mathrm{m}$ to form the silicon nanowires.

\section{Results and Discussion}

For the $\mathrm{ZnO}$ nanowire array after sideways PLD at room temperature, the NWs were very bent. However, as the temperature was increased, the bending angle became smaller. At a temperature above $120{ }^{\circ} \mathrm{C}$, the NWs remained almost straight after sideways PLD. And as the temperature continuously increased to $300{ }^{\circ} \mathrm{C}$, the NWs remained as hexagonal pillars with a single crystal structure after sideways PLD. This result confirmed that the bending phenomenon is not caused by thermal stress because a thermal stress effect should be more significant if the deposition process is carried out at a higher 


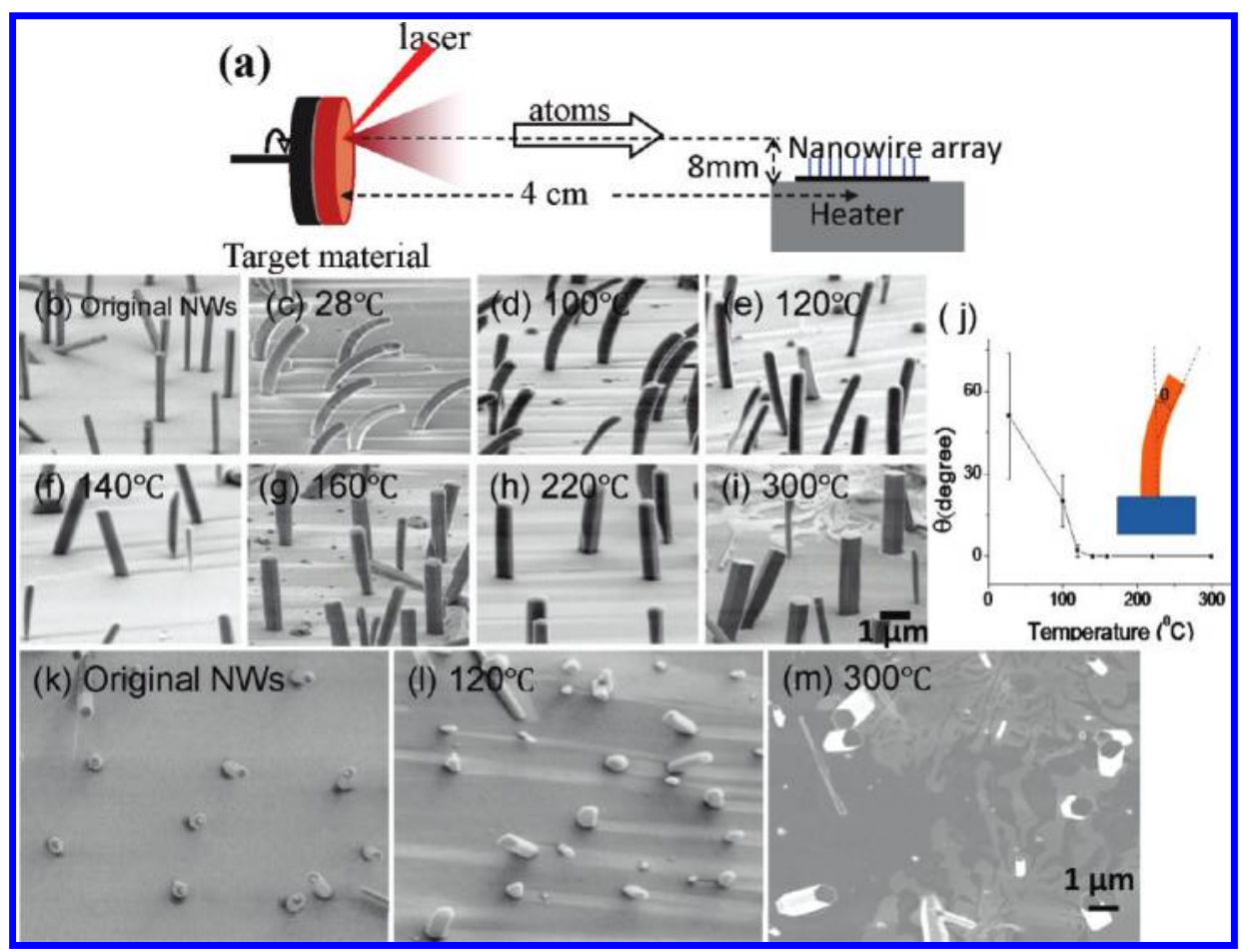

Figure 1. (a) Schematic of the setup of sideways PLD. The substrate was flat without tilting. (b) Side view (tilt angle $=60^{\circ}$ ) SEM image of the original $\mathrm{ZnO}$ nanowires. (c-i) Side view SEM images of nanowires after depositing ZnO from the left side at different temperatures, respectively. (j) Measured bending angle of the wires as a function of temperature. $(k-m)$ Top view SEM images of original NWs, samples deposited at 120 ${ }^{\circ} \mathrm{C}$ and at $300{ }^{\circ} \mathrm{C}$, respectively.

temperature. The bending is more likely to be caused by the stress accumulation at the side surface. The high energy incoming atoms may implant into the surface of one side of the NWs and induce strain. Since the diffusion of atoms is rather strong at higher temperature, they tend to migrate to lower energy positions to release the local strain, which may reduce the degree of NW bending. Therefore, the bending phenomenon is likely to be observed at lower temperatures. At $300{ }^{\circ} \mathrm{C}$, the new incoming atoms will have enough energy to epitaxially crystallize on the surface of the NWs to form thicker NWs and keep the hexagonal shape. Considering that $\mathrm{ZnO}$ has a bulk melting point of $1975^{\circ} \mathrm{C}$, surface diffusion is possible at low temperature. ${ }^{26}$ Our result indicates that surface diffusion on the NWs can occur at a temperature even lower than $300{ }^{\circ} \mathrm{C}$.

Another thing that should be noticed in the SEM images is the change of the "shadows" beside the NWs. These shadows were formed because the NWs blocked some of the incoming atoms. As the temperature was increased, the shadows became shallower. At $300{ }^{\circ} \mathrm{C}$, there was almost no visible shadow. And on the other hand, the NWs fabricated at $300{ }^{\circ} \mathrm{C}$ were much thicker than other samples. This phenomenon may be because the $\mathrm{ZnO}$ atoms migrated from the surface of the $\mathrm{Au}$ substrate to the $\mathrm{ZnO}$ NWs.

In the XRD patterns (Figure 2a), the shape of the $\mathrm{ZnO}(0002)$ peak suggests that the composite NW is composed of a nanocrystalline shell (not uniform in thickness) and a main crystalline body; the former gives a broader peak, the latter gives a sharp peak, and the two peaks are located at different positions possibly due to a strain-induced lattice expansion of the nanocrystallites. At room temperature, the XRD intensity from the nanocrystallite shell was large. That is because most atoms at the surface did not have enough energy to migrate and just remained as deposited. The peak from the crystalline body was relatively broad compared with that at higher temperature because the NWs were very bent and the strain inside the NWs was nonuniform. These two kinds of peaks merged together and formed one asymmetric peak, as shown in the XRD spectrum. As the temperature was increased, the peak intensity of the nanocrystallites became smaller, which means that more atoms had enough energy to migrate and the deposited layer became more crystalline. The peak from the crystalline body became sharper (Figure $2 b$ ) because the NWs were straightened and part of the strain was released.

By comparing the position of the peak from the crystalline body with that of the original NWs, the peak was shifted to a lower angle after PLD, which means there was a tensile strain inside the NWs, in agreement with the atom implantation mechanism. Even at $300{ }^{\circ} \mathrm{C}$, the strain still could not be totally released.

Evaporator, sputter, and PLD are three most commonly used methods to deposit various materials. By using different methods, the energy of the atoms from the source materials is very different. In the evaporator, the average kinetic energy of the incoming atoms can be simply calculated using the Boltzmann equation $\left(E_{\mathrm{k}}=3 / 2 k_{\mathrm{B}} T\right)$. At $3000 \mathrm{~K}$, the average kinetic energy is only $0.38 \mathrm{eV}$. For the sputter technique, the energy of the incident particles into $\mathrm{ZnO}$ is the energy when they were reflected from the raw target material minus that lost in collisions with the working gas before striking the ZnO. Generally, the average kinetic energy of the incoming atoms is on the order of several electron volts. ${ }^{27}$ For PLD, the typical velocity of the plume (consisting of atoms from the target) is $10^{6} \mathrm{~cm} / \mathrm{s} .{ }^{28}$ At that velocity, for Au atoms, the kinetic energy is $98 \mathrm{eV}$. Higher energy atoms are more likely to implant into the surface, so the bending phenomenon should be more obvious in PLD than in sputter and evaporator deposited NWs. We found that after PLD, the microwire was obviously bent downward (Figure 3a). After sputter, the wire was slightly bent downward (Figure 3b). After evaporator deposition, however, the wire was found to be bent upward a tiny bit (Figure 3c). The shape change after 


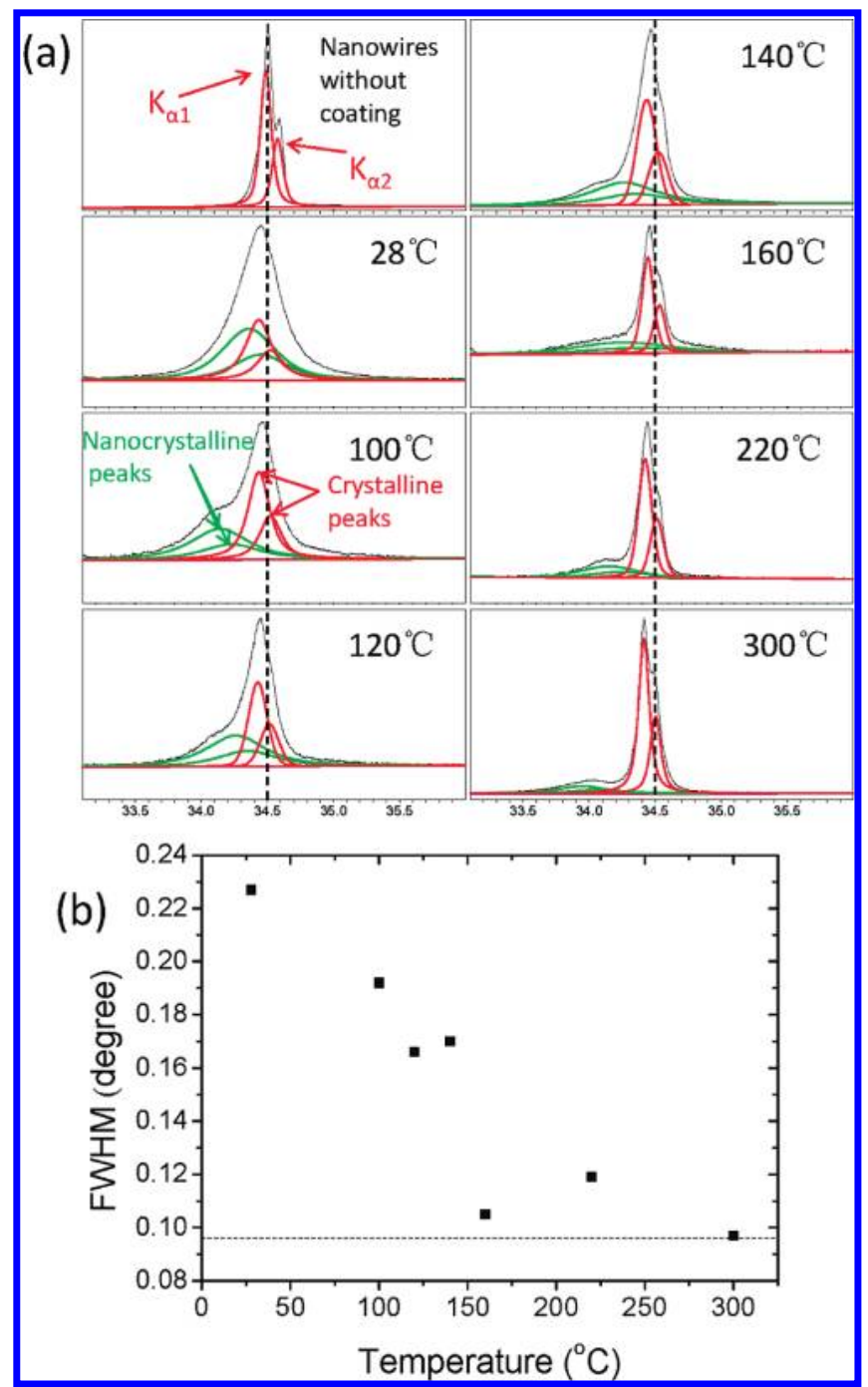

Figure 2. (a) XRD analysis of the (0002) peak of original $\mathrm{ZnO}$ nanowires and $\mathrm{ZnO}$ nanowires after PLD at different temperatures. The vertical dashed line indicates the peak position of the original $\mathrm{ZnO}$ nanowires. (b) The width of the crystalline peak from samples prepared at different temperatures. The horizontal dashed line indicates the fwhm of the original $\mathrm{ZnO}$ nanowires.

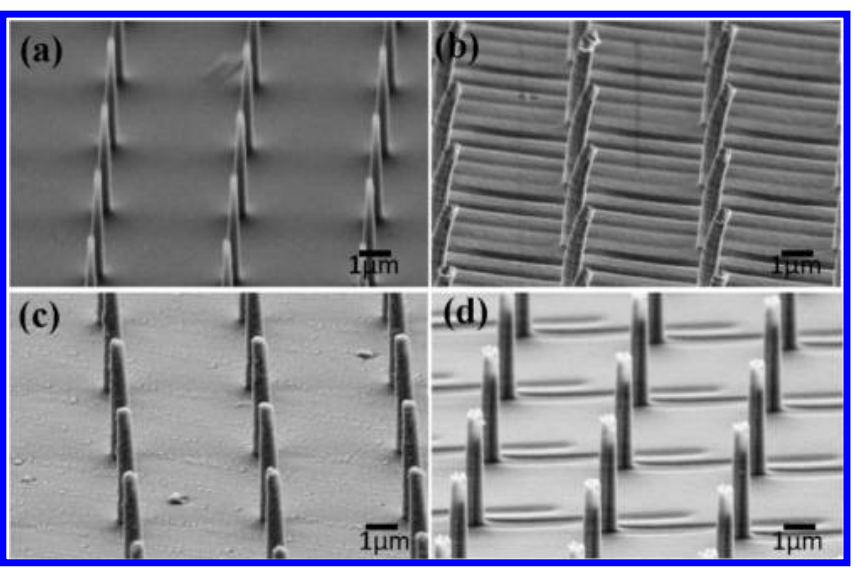

Figure 4. SEM images of original Si nanowires (a) and Si nanowires after depositing Ti on the left sidewall using PLD (b), sputter (c), and evaporator $(\mathrm{d})$.

evaporator deposition may be due to thermal stress. The sample holder was hot in the evaporation process, and when it was cooled down to room temperature, the Au side would contract more than the $\mathrm{ZnO}$ side because the thermal expansion coefficient of $\mathrm{Au}$ is larger than that of $\mathrm{ZnO}$.

Similar results can also be found for $\mathrm{Si}$ NWs. Ti was deposited on the side wall of the Si NWs using different methods (Figure 4). Only PLD can make the NWs bend significantly. The bending direction is also the same as the incoming direction of the incoming atom plume.

\section{Conclusion}

Room temperature PLD was found to be the most efficient method to bend NWs. The bending is probably caused by the ion implantation into the surface layers. Temperature can influence the bending angle at low temperatures (below 120 $\left.{ }^{\circ} \mathrm{C}\right)$. For sputtering, because the energy of the atoms is smaller, the bending angle of the NWs is much smaller than the PLDdeposited ones. For the evaporator method, the energy of the atoms is even smaller than that in the sputter method. The ion implantation effect is negligible. The thermal stress will cause the NWs to bend a tiny bit in the opposite direction. Considering that the physical properties of NWs are closely related to the

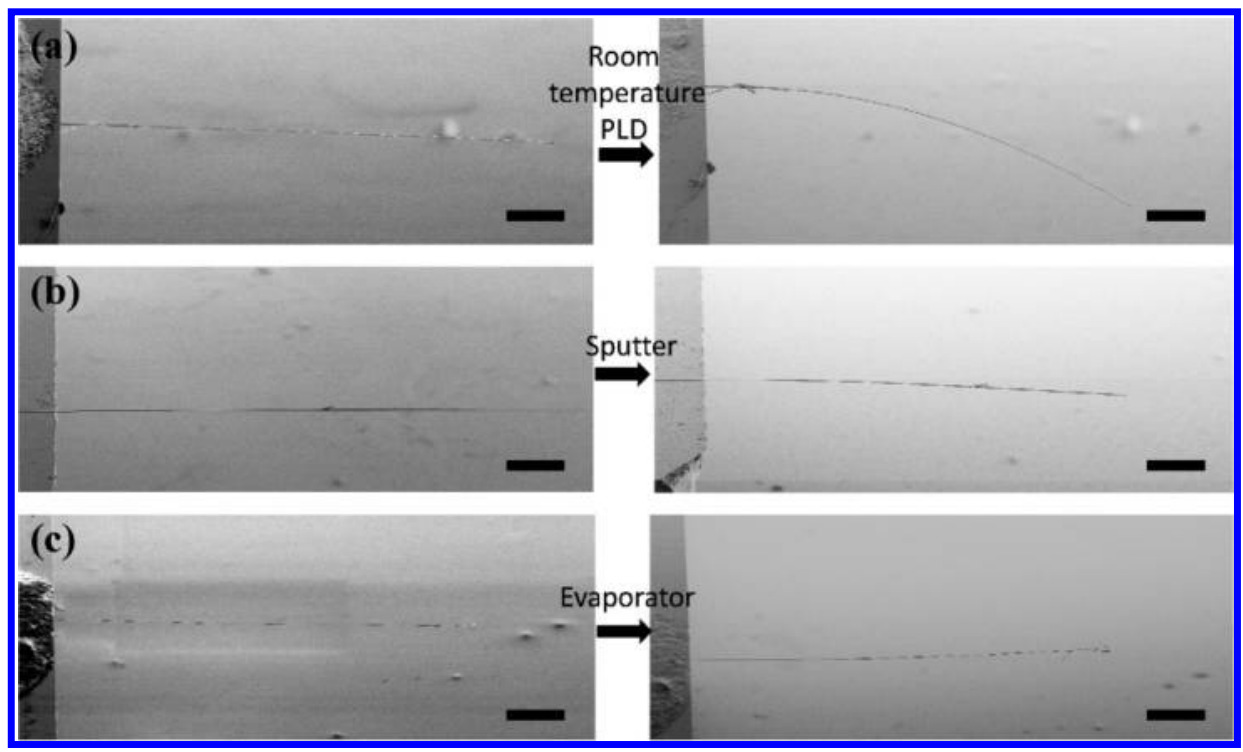

Figure 3. Shape evolution of free-standing $\mathrm{ZnO}$ microwires after depositing Au from the top using different deposition methods. The SEM images were taken at a tilt angle of $45^{\circ}$. 
shape and strain, this method adds a new tool for the production of nanodevices.

Acknowledgment. This research was supported by NSF, BES DOE, and the Air Force. Z.L.W. thanks the support from the WCU program, UNIST, Korea. Y.S. thanks the fellowship support by the China Scholarship Council (CSC) (No. 2009621143) for carrying out the research at Georgia Tech.

\section{References and Notes}

(1) Dong, L.; Zhang, L.; Bell, D. J.; Grützmacher, D.; Nelson, B. J. J. Phys. Conf. Ser. 2007, 61, 257.

(2) Prinz, V. Y. Microelectron. Eng. 2003, 69, 466.

(3) Nakakubo, T.; Shimoyama, I. Sens. Actuators 2000, 83, 161.

(4) Syms, R. R. A.; Yeatman, E. M.; Bright, V. M.; Whitesides, G. M. J. Microelectromech. Syst. 2003, 12, 387.

(5) Tian, B.; Cohen-Karni, T.; Qing, Q.; Duan, X.; Xie, P.; Lieber, C. M. Science 2010, 329, 830.

(6) Wang, Z. L. Mat. Sci. Eng. R 2009, 64, 33.

(7) Xia, Y.; Yang, P.; Sun, Y.; Wu, Y.; Mayers, B.; Gates, B.; Yin, Y.; Kim, F.; Yan, H. Adv. Mater. 2003, 15, 353.

(8) Morales, A. M.; Lieber, C. M. Science 1998, 279, 208.

(9) A Special Issue on Carbon Nanotubes. Acc. Chem. Res. 2002, 36, 997.

(10) Pan, Z. W.; Dai, Z. R.; Wang, Z. L. Science 2001, $291,1947$.

(11) Gao, P. X.; Ding, Y.; Mai, W.; Hughes, W. L.; Lao, C. S.; Wang, Z. L. Science 2005, 309, 1700.

(12) Zhang, L.; Deckhardt, E.; Weber, A.; Schonenberger, C.; Grutzmacher, D. Nanotechnology 2005, 16, 655.
(13) Kong, X. Y.; Ding, Y.; Yang, R. S.; Wang, Z. L. Science 2004, 303,1348

(14) Chen, Z.; Shan, Z.; Cao, M. S.; Lu, L.; Mao, S. X. Nanotechnology 2004, 15,365 .

(15) Kim, T.-I.; Suh, K. Y. Soft Mater. 2009, 5, 4131.

(16) Chu, K.-H.; Xiao, R.; Wang, E. N. Nat. Mater. 2010, 9, 413.

(17) He, J. H.; Hsin, C. L.; Liu, J.; Chen, L. J.; Wang, Z. L. Adv. Mater. 2007, 19, 781 .

(18) Gao, Z.; Zhou, J.; Gu, Y.; Fei, P.; Hao, Y.; Bao, G.; Wang, Z. L. J. Appl. Phvs. 2009, 105, 113707.

(19) Han, X.; Kou, L.; Lang, X.; Xia, J.; Wang, N.; Qin, R.; Lu, J.; Xu, J.; Liao, Z.; Zhang, X.; Shan, X.; Song, X.; Gao, J.; Guo, W.; Yu, D. $\underline{A d v}$. Mater. 2009, 21, 4937.

(20) Xue, H.; Pan, N.; Li, M.; Wu, Y.; Wang, X.; Hou, J. G. Nanotechnology 2010, 21, 215701.

(21) Whittaker, L.; Jaye, C.; Fu, Z.; Fischer, D. A.; Banerjee, S. J. Am. Chem. Soc. 2009, 131, 8884.

(22) Cao, J.; Ertekin, E.; Srinivasan, V.; Fan, W.; Huang, S.; Zheng, H.; Yim, J. W. L.; Khanal, D. R.; Ogletree, D. F.; Grossman, J. C.; Wu, J. Nat. Nanotechnol. 2009, 4, 732 .

(23) Shen, Y.; Hong, J.-I.; Xu, S.; Lin, S.; Fang, H.; Zhang, S.; Ding, Y.; Snyder, R. L.; Wang, Z. L. Adv. Funct. Mater. 2010, $20,703$.

(24) Xu, S.; Lao, C. S.; Weintraub, B.; Wang, Z. L. J. Mater. Res. 2008, $23,2072$.

(25) Laermer, F.; Urban, A. Microelectron. Eng. 2003, 67-68, 349.

(26) Wang, Z. L.; Petroski, J.; Green, T.; El-Sayed, M. A. J. Phys. Chem. $\underline{B}$ 1998, 102, 6145 .

(27) Muller, K.-H. J. Appl. Phvs. 1987, 62, 1796.

(28) Singh, R. K.; Narayan, J. Phvs. Rev. B 1990, 41, 8843.

JP108691Y 